Pacific

Journal of

Mathematics

PHRAGMÈN-LINDELÖF THEOREM FOR MINIMAL SURFACE EQUATIONS IN HIGHER DIMENSIONS

Chun-Chung Hsieh, Jenn-Fang Hwang, and Fei-Tsen Liang 


\title{
PHRAGMÈN-LINDELÖF THEOREM FOR MINIMAL SURFACE EQUATIONS IN HIGHER DIMENSIONS
}

\author{
Chun-Chung Hsieh, Jenn-Fang Hwang, and Fei-Tsen Liang
}

Here we prove that if $u$ satisfies the minimal surface equation in an unbounded domain which is properly contained in a half space of $\mathbb{R}^{n}$, with $n \geq 2$, then the growth rate of $u$ is of the same order as that of the shape of $\Omega$ and the boundary value of $u$.

\section{Introduction.}

Consider the minimal surface equation

$$
\operatorname{div} T u=0,
$$

where

$$
T u=\frac{\nabla u}{\sqrt{1+|\nabla u|^{2}}} \quad \text { and } \quad \nabla u=\left(u_{x_{1}}, \ldots, u_{x_{n}}\right) .
$$

In 1965, Nitsche [7] announced the following result: "Let $\Omega_{\alpha} \subset \mathbb{R}^{2}$ be a sector with angle $0<\alpha<\pi$. If $u$ satisfies the minimal surface equation with vanishing boundary value in $\Omega_{\alpha}$, then $u \equiv 0$ ". Hwang extends this result in $[4],[5],[6]$ and proves that, in an unbounded domain $\Omega$ properly contained in the half plane in $\mathbb{R}^{2}$, if $u$ satisfies the minimal surface equation, then, the growth property of $u$ is determined completely by the shape of $\Omega$ and the boundary value of $u$. In this respect, the Phragmèn-Lindelöf theorem for the minimal surface equation is better than that for the Laplace equation. (Indeed, if $u$ satisfies the Laplace equation in an unbounded domain $\Omega$, the growth property of $u$ cannot be determined completely by the shape of $\Omega$ and the boundary data of $u$ alone (cf. [10]).)

The purpose of this paper is to generalize the two-dimensional PhragmènLindelöf theorems in [4], [5] and [6], to higher dimensions. In $\S 2$, we review the statements of the Phragmèn-Lindelöf theorem of $[4],[5]$ and $[6]$. The higher-dimensional version is similar in content, but proof is different. In $\S 3$, based on an argument of [2], we established the suitable comparison principle. In $\S 4$, we compute the mean curvature of our comparison function, and use it to finish the proof of our main theorems in $\S 5$. 


\section{Preliminary.}

The main purpose of this paper is to generalize the two-dimensional Phragmèn-Lindelöf theorem in $[4],[5],[6]$ to higher dimensions. We may, first of all, recall some results in these papers and consider functions

$$
f:[0, \infty) \rightarrow[0, \infty), f \in C^{2}([0, \infty)), f^{\prime} \equiv \frac{d f(y)}{d y}>0,
$$

from which we define

$$
p(f)=1-\frac{f f^{\prime \prime}}{\left(f^{\prime}\right)^{2}} .
$$

In particular, for $f(y)=y^{m}, m$ being a positive constant, we have

$$
p(f)=\frac{1}{m},
$$

which is precisely the reciprocal of the order of $f$, while for $f(y)=e^{y}$, we have

$$
p(f)=0
$$

moreover, in case $f$ grows faster than the exponential function, we can assume $p(f) \geq-\epsilon$ for some small positive constant $\epsilon$, essentially (cf. [5, Remark 2.7]). Accordingly, we may proceed to solve the ordinary differential equation in $[-1,1]$

$$
(1-p(f))\left(h-t h^{\prime}\right)\left(1+{h^{\prime}}^{2}\right)+h^{\prime \prime}\left(h^{2}+t^{2}\right)=0
$$

with initial values

$$
h(-1)=0 \quad \text { and } \quad h^{\prime}(-1)=\tan \left((1-p(f)) \frac{\pi}{2}\right),
$$

and then denote its solution, if exists, by $h_{m}$ if $f(y)=y^{m}$ (and hence $p(f)=\frac{1}{m}$ ), and by $h_{\infty}$ if $f(y)=e^{y}$ (and hence $p(f)=0$ ). In general, $(*)$ and $(* *)$ cannot be solved explicitly; but, for some specific $m$, its solution can be written out explicitly. For example, we have

$$
h_{2}=\frac{1-t^{2}}{2}
$$

and also

$$
h_{\infty}=\sqrt{1-t^{2}}
$$

It is useful to know some interesting properties of $h_{m}, 0<m \leq \infty$, in the following:

Lemma 1 ([6]). For $1<m, m^{\prime} \leq \infty$ and $t \in(-1,1)$, then we have

$$
h_{m}(t)>h_{m^{\prime}}(t), \quad \text { whenever } m>m^{\prime},
$$

and

$$
h_{m}(t)<h_{m}\left(t^{\prime}\right), \quad \text { whenever }|t|>\left|t^{\prime}\right|
$$


The Phragmèn-Lindelöf theorems in [5], [6] can now be formulated as follows.

Theorem 2. Let $\Omega \subseteq\left\{(x, y) \in \mathbb{R}^{2} \mid \quad-a y^{m}<x<a y^{m}, y>0\right\} \subseteq \mathbb{R}^{2}$ be an unbounded domain, where $a$ and $m$ are positive constants, $m \geq 1$. Let $u \in C^{2}(\Omega) \cap C^{0}(\bar{\Omega})$ and suppose that

$$
\begin{cases}\operatorname{div} T u \geq 0 & \text { in } \Omega \\ u \leq a y^{m} h_{m}\left(\frac{x}{a y^{m}}\right) & \text { on } \partial \Omega .\end{cases}
$$

Then we have $u \leq a y^{m} h_{m}\left(\frac{x}{a y^{m}}\right) \leq a y^{m} h_{\infty}\left(\frac{x}{a y^{m}}\right)=\sqrt{a^{2} y^{2 m}-x^{2}}$ in $\Omega$.

Theorem 2*. Let $\Omega \subseteq\left\{(x, y) \in \mathbb{R}^{2} \mid-a e^{b y}<x<a e^{b y}, y>0\right\}$ be an unbounded domain where $a, b$ are positive constants. Let $u \in C^{2}(\Omega) \cap C^{0}(\bar{\Omega})$ and suppose that

$$
\begin{cases}\operatorname{div} T u \geq 0 & \text { in } \Omega \\ u \leq \sqrt{a^{2} e^{2 b y}-x^{2}} & \text { on } \partial \Omega .\end{cases}
$$

Then we have $u \leq \sqrt{a^{2} e^{2 b y}-x^{2}}$ in $\Omega$.

Theorem 3. Let $f \in C^{2}([0, \infty)), f>0, f^{\prime}>0$ in $(0, \infty)$ and $p(f) \geq p_{0}$, where $p_{0}$ is a negative constant, and let $f_{1} \in C^{0}([0, \infty))$ and $f_{1}>0$ in $(0, \infty)$. For a given unbounded open domain

$$
\Omega \subset\left\{(x, y) \in \mathbb{R}^{2} \mid-f_{1}(y)<x<f_{1}(y), y>0\right\},
$$

and $u \in C^{2}(\Omega) \cap C^{0}(\bar{\Omega})$ with

$$
\begin{cases}\operatorname{div} T u \geq 0 & \text { in } \Omega \\ u \leq a \sqrt{f^{2}-x^{2}} & \text { on } \partial \Omega,\end{cases}
$$

where $f^{2} \geq \frac{\left(a^{2}-1\right)\left(2-p_{0}\right)}{\left(a^{2}-\left(1-p_{0}\right)\right)} f_{1}^{2}$ and $a$ is a positive constant satisfying

$$
a^{2}-1+p_{0}>0 \text {. }
$$

Then, we have

$$
u \leq a \sqrt{f^{2}-x^{2}} \quad \text { in } \quad \Omega .
$$

Remark. In Theorem 3, since $p_{0}<0$ and $a>0$, we have

$$
\frac{\left(a^{2}-1\right)\left(2-p_{0}\right)}{\left(a^{2}-\left(1-p_{0}\right)\right)}=\left(\frac{a^{2}-1}{a^{2}-\left(1-p_{0}\right)}\right)\left(2-p_{0}\right)>2 .
$$

Thus, in case $u \leq 0$ on $\partial \Omega$, our estimates are not good enough since we use worse boundary conditions, whereas the best estimates remain unknown.

These theorems will be generalized to higher dimensions in $\S 5$. 


\section{A comparison principle.}

To establish the higher-dimensional Phragmèn-Lindelöf theorem, we shall need the following comparison principle.

Lemma 4. Let $\Omega$ be an unbounded domain in $\mathbb{R}^{n}$, and let $u, v \in C^{2}(\Omega) \cap$ $C^{0}(\bar{\Omega})$. Suppose that

$$
\begin{cases}\operatorname{div} T u-\operatorname{div} T v \geq C & \text { in } \Omega, \\ u \leq v & \text { on } \partial \Omega,\end{cases}
$$

where $C$ is a positive constant. Then we have $u \leq v$ in $\Omega$.

Proof. The idea of proof is analogous to that of [2].

Suppose that this lemma fails to hold. There then exists a positive constant $\epsilon$ such that

$$
\Omega^{\prime}=\{x \in \Omega \mid u(x)>v(x)+\epsilon\}
$$

is not empty; by Sard's theorem, we may further assume that $\partial \Omega^{\prime} \cap \Omega$ is smooth. For every $R>0$, set

$$
\begin{aligned}
& B_{R}=\left\{x \in \mathbb{R}^{n}|\quad| x \mid<R\right\}, \\
& \Omega_{R}=B_{R} \cap \Omega^{\prime}, \\
& \Gamma_{R}=\partial B_{R} \cap \partial \Omega_{R},
\end{aligned}
$$

and

$$
\left|\Gamma_{R}\right|=\text { the Hausdorff }(n-1)-\text { dimensional measure of } \Gamma_{R} .
$$

Also, let

$$
\begin{aligned}
g(R) & =\oint_{\partial \Omega_{R}} \tan ^{-1}(u-v-\epsilon)(T u-T v) \cdot \nu \\
& =\int_{\Gamma_{R}} \tan ^{-1}(u-v-\epsilon)(T u-T v) \cdot \nu
\end{aligned}
$$

where $\nu$ is the unit outward normal of $\partial \Omega_{R}$.

Then we have

$$
\begin{aligned}
g(R)= & \iint_{\Omega_{R}} \frac{(\nabla u-\nabla v) \cdot(T u-T v)}{1+(u-v-\epsilon)^{2}} \\
& +\iint_{\Omega_{R}} \tan ^{-1}(u-v-\epsilon)(\operatorname{div} T u-\operatorname{div} T v) .
\end{aligned}
$$

Since the integrand of the right-hand side of (1) is nonnegative, Fubini's theorem tells us that $g^{\prime}(R)$ exists for almost all $R>0$, and whenever it 
exists, we have, by (2),

$$
\begin{aligned}
g^{\prime}(R)= & \int_{\Gamma_{R}} \frac{(\nabla u-\nabla v) \cdot(T u-T v)}{1+(u-v-\epsilon)^{2}} \\
& +\int_{\Gamma_{R}} \tan ^{-1}(u-v-\epsilon)(\operatorname{div} T u-\operatorname{div} T v) \\
\geq & C \int_{\Gamma_{R}} \tan ^{-1}(u-v-\epsilon), \quad \text { (by assumption) } \\
\geq & \frac{C}{2} \int_{\Gamma_{R}} \tan ^{-1}(u-v-\epsilon)|T u-T v|, \\
\geq & \frac{C}{2} g .
\end{aligned}
$$

Since $g$ is an increasing function of $R$ and $g \geq 0$, it is easy to see that Lemma 4 holds in the case that $g \equiv 0$. If, on the other hand, $g \not \equiv 0$, there would exist a positive constant $R_{0}$ such that $g(R)>0$ for all $R \geq R_{0}$, and hence, for every $R>R_{0}$, in virtue of (3)

$$
\int_{R_{0}}^{R} \frac{g^{\prime}(r)}{g(r)} d r \geq \frac{C}{2}\left(R-R_{0}\right),
$$

i.e.,

$$
\left.\log g(r)\right|_{R_{0}} ^{R} \geq \frac{C}{2}\left(R-R_{0}\right),
$$

and therefore,

$$
g(R) \geq g\left(R_{0}\right) e^{\frac{c}{2}\left(R-R_{0}\right)} .
$$

However, we have, by (1)

$$
g(R) \leq \int_{\Gamma_{R}} \frac{\pi}{2} \cdot 2 \leq \pi\left|\Gamma_{R}\right| ;
$$

since $\Gamma_{R} \subset \partial B_{R}$, this yields a positive constant $C_{1}$ completely determined by $n$ such that

$$
g(R) \leq C_{1} R^{n-1},
$$

which contradicts (4) and yields the truth of Lemma 4.

Remark. The above proof works well and so the lemma is valid if $v=+\infty$ on some parts of $\partial \Omega$. 


\section{An estimation of the growth of solutions.}

Henceforth, we will denote $\Omega$ as an unbounded domain in $\mathbb{R}^{n}, n \geq 2$, such that, for some $f \in C^{2}([0, \infty)), f>0, f^{\prime}>0$ and $f^{\prime \prime}>0$ in $(0, \infty)$, we have

$$
\Omega \subset\left\{(x, y) \in \mathbb{R}^{2} \mid-f(y)<x<f(y), y>o\right\} \times \mathbb{R}^{n-2} \subset \mathbb{R}^{n} .
$$

We shall extend the results in $\S 2$ to such a domain $\Omega$.

First, for every positive constant $y_{0}$, since $f>0, f^{\prime}>0$ and $f^{\prime \prime}>0$ in $(0, \infty)$, it is easy to see that there exists a positive constant $\delta_{1}$, depending on $y_{0}$, such that $\left\{(f(y), y) \in \mathbb{R}^{2} \mid \quad y>0\right\} \cap\left\{(x, y) \in \mathbb{R}^{2} \mid \quad y_{0}-y+\frac{\delta_{1}}{2} x^{2}=0\right\}$ has exactly one point. And also, $\left\{(f(y), y) \in \mathbb{R}^{2} \mid \quad y>0\right\} \cap\left\{(x, y) \in \mathbb{R}^{2} \mid y_{0}-\right.$ $\left.y+\frac{\delta}{2} x^{2}=0\right\}$ has exactly two points, say $\left(f\left(y_{1}\right), y_{1}\right)$ and $\left(f\left(y_{2}\right), y_{2}\right)$ with $0<y_{1}<y_{2}$, for all $\delta$ with $0<\delta<\delta_{1}$. In general, we have $y_{1}=y_{1}\left(y_{0}, \delta\right)$, $y_{2}=y_{2}\left(y_{0}, \delta\right)$ and also $\lim _{\delta \rightarrow 0} y_{1}\left(y_{0}, \delta\right)=y_{0}$. From now on, we always assume that the positive constant $\delta$ is less than the above $\delta_{1}$.

To apply Lemma 4 to estimate the speed of growth of solutions in $\Omega$, we may consider comparison functions of the following form

$$
F_{y_{0}, \delta}=\frac{A\left(f^{2}(y)-x^{2}\right)^{\frac{1}{2}}}{y_{0}-y+\frac{\delta}{2} x^{2}},
$$

which is defined on

$$
\Omega_{y_{0}, \delta}=\Omega \cap\left(\left\{(x, y) \in \mathbb{R}^{2} \mid y_{0}-y+\frac{\delta}{2} x^{2}>0,0<y<y_{1}\right\} \times \mathbb{R}^{n-2}\right),
$$

where $\delta, y_{0}$, and $A$ are positive constants. We first proceed to calculate the mean curvature of $F_{y_{0}, \delta}$. For convenience of computation, we may set

$$
F=A \cdot P^{\frac{1}{2}} Q^{-1},
$$

where $P=f^{2}(y)-x^{2}$ and $Q=y_{0}-y+\frac{\delta}{2} x^{2}$. We observe that

$$
\begin{aligned}
\operatorname{div} T F & =\frac{\left(1+F_{x}^{2}\right) F_{y y}-2 F_{x} F_{y} F_{x y}+\left(1+F_{y}^{2}\right) F_{x x}}{\left(1+F_{x}^{2}+F_{y}^{2}\right)^{\frac{3}{2}}} \\
& =\frac{\left(\frac{1}{F^{2}}+\frac{F_{x}^{2}}{F^{2}}\right) \frac{F_{y y}}{F}-2 \frac{F_{x}}{F} \frac{F_{y}}{F} \frac{F_{x y}}{F}+\left(\frac{1}{F^{2}}+\frac{F_{y}^{2}}{F^{2}}\right) \frac{F_{x x}}{F}}{\left(\frac{1}{F^{2}}+\left(\frac{F_{x}}{F}\right)^{2}+\left(\frac{F_{y}}{F}\right)^{2}\right)^{\frac{3}{2}}} .
\end{aligned}
$$

Denoting

$$
\mathrm{I}=\frac{F_{x}^{2}}{F^{2}} \frac{F_{y y}}{F}+\frac{F_{y}^{2}}{F^{2}} \frac{F_{x x}}{F}-2 \frac{F_{x}}{F} \frac{F_{y}}{F} \frac{F_{x y}}{F}
$$

and

$$
\mathrm{II}=\frac{F_{x x}}{F^{3}}+\frac{F_{y y}}{F^{3}},
$$


we note that the numerator in (5) is the sum of these two expressions and we shall treat them seperately. For the first expression, we have

$$
\begin{aligned}
\mathrm{I}= & \frac{F_{x}^{2}}{F^{2}}\left(\partial_{y}\left(\frac{F_{y}}{F}\right)+\left(\frac{F_{y}}{F}\right)^{2}\right)+\frac{F_{y}^{2}}{F^{2}}\left(\partial_{x}\left(\frac{F_{x}}{F}\right)+\left(\frac{F_{x}}{F}\right)^{2}\right) \\
& -2 \frac{F_{x}}{F} \frac{F_{y}}{F}\left[\partial_{x}\left(\frac{F_{y}}{F}\right)+\frac{F_{x} F_{y}}{F^{2}}\right] \\
= & \frac{F_{x}^{2}}{F^{2}}\left(\partial_{y}\left(\frac{F_{y}}{F}\right)\right)+\frac{F_{y}^{2}}{F^{2}}\left(\partial_{x}\left(\frac{F_{x}}{F}\right)\right)-2 \frac{F_{x} F_{y}}{F^{2}}\left(\partial_{x}\left(\frac{F_{y}}{F}\right)\right) \\
= & \mathrm{I}^{*}+\mathrm{I}^{* *}
\end{aligned}
$$

where

$$
\begin{aligned}
\mathrm{I}^{*}= & \frac{F_{x}^{2}}{F^{2}}\left(-\frac{1}{2} \frac{P_{y}^{2}}{P^{2}}+\frac{Q_{y}^{2}}{Q^{2}}\right)+\frac{F_{y}^{2}}{F^{2}}\left(-\frac{1}{2} \frac{P_{x}^{2}}{P^{2}}+\frac{Q_{x}^{2}}{Q^{2}}\right) \\
& -2 \frac{F_{x} F_{y}}{F^{2}}\left(-\frac{1}{2} \frac{P_{x} P_{y}}{P^{2}}+\frac{Q_{x} Q_{y}}{Q^{2}}\right),
\end{aligned}
$$

and

$$
\begin{aligned}
\mathrm{I}^{* *}= & \frac{F_{x}^{2}}{F^{2}}\left(\frac{1}{2} \frac{P_{y y}}{P}-\frac{Q_{y y}}{Q}\right)+\frac{F_{y}^{2}}{F^{2}}\left(\frac{1}{2} \frac{P_{x x}}{P}-\frac{Q_{x x}}{Q}\right) \\
& -2 \frac{F_{x} F_{y}}{F^{2}}\left(\frac{1}{2} \frac{P_{x y}}{P}-\frac{Q_{x y}}{Q}\right) .
\end{aligned}
$$

By a direct computation,

$$
\mathrm{I}^{*}=\frac{-1}{4} \frac{1}{P^{2} Q^{2}}\left(P_{y} Q_{x}-P_{x} Q_{y}\right)^{2},
$$

while

$$
\begin{aligned}
\mathrm{I}^{* *}= & \left(\frac{1}{2} \frac{P_{x}}{P}-\frac{Q_{x}}{Q}\right)^{2}\left(\frac{1}{2} \frac{P_{y y}}{P}-\frac{Q_{y y}}{Q}\right) \\
& +\left(\frac{1}{2} \frac{P_{y}}{P}-\frac{Q_{y}}{Q}\right)^{2}\left(\frac{1}{2} \frac{P_{x x}}{P}-\frac{Q_{x x}}{Q}\right) \\
& -2\left(\frac{1}{2} \frac{P_{x}}{P}-\frac{Q_{x}}{Q}\right)\left(\frac{1}{2} \frac{P_{y}}{P}-\frac{Q_{y}}{Q}\right)\left(\frac{1}{2} \frac{P_{x y}}{P}-\frac{Q_{x y}}{Q}\right) .
\end{aligned}
$$

Thus, in particular, we have

$$
\mathrm{I}^{*} \leq 0
$$

As for I** and II, we recall that

$$
P=f^{2}(y)-x^{2} \quad \text { and } \quad Q=y_{0}-y+\frac{\delta}{2} x^{2},
$$


and hence

$$
P_{x}=-2 x, \quad P_{y}=2 f^{\prime} f, \quad Q_{x}=\delta x, \quad Q_{y}=-1 ;
$$

moreover

$$
P_{x x}=-2, \quad P_{x y}=0, \quad P_{y y}=2\left(f^{\prime \prime} f+f^{\prime} f^{\prime}\right)
$$

and

$$
Q_{x x}=\delta, \quad Q_{x y}=0, \quad Q_{y y}=0 .
$$

Thus, we have

$$
\begin{aligned}
\mathrm{I}^{* *}= & \frac{1}{P^{3}}\left[x^{2}\left(f^{\prime \prime} f+f^{\prime 2}\right)-f^{2} f^{\prime 2}\right] \\
& +\frac{(-2)}{P^{2} Q}\left[-\delta x^{2}\left(f^{\prime \prime} f+f^{\prime 2}\right)+f^{\prime} f+\frac{\delta}{2} f^{2} f^{\prime 2}\right] \\
& +\frac{1}{P Q^{2}}\left[\delta^{2} x^{2}\left(f^{\prime \prime} f+f^{\prime 2}\right)-1-2 \delta f f^{\prime}\right]-\delta Q^{-3},
\end{aligned}
$$

and also

$$
\begin{aligned}
\mathrm{II}= & \frac{Q^{2}}{A^{2} P}\left(\partial_{x}\left(\frac{F_{x}}{F}\right)+\frac{F_{x}^{2}}{F^{2}}+\partial_{y}\left(\frac{F_{y}}{F}\right)+\frac{F_{y}^{2}}{F^{2}}\right) \\
= & \frac{Q^{2}}{A^{2} P}\left[\partial_{x}\left(\frac{1}{2} \frac{P_{x}}{P}-\frac{Q_{x}}{Q}\right)+\partial_{y}\left(\frac{1}{2} \frac{P_{y}}{P}-\frac{Q_{y}}{Q}\right)\right. \\
& \left.+\left(\frac{1}{2} \frac{P_{x}}{P}-\frac{Q_{x}}{Q}\right)^{2}+\left(\frac{1}{2} \frac{P_{y}}{P}-\frac{Q_{y}}{Q}\right)^{2}\right] \\
= & \frac{Q^{2}}{A^{2}}\left\{\frac{1}{P^{3}}\left[f^{2}\left(f^{\prime \prime} f-1\right)-x^{2}\left(f f^{\prime \prime}+f^{\prime 2}\right)\right]\right. \\
& \left.+\frac{1}{P^{2} Q}\left(2 f f^{\prime}-\delta f^{2}+3 \delta x^{2}\right)+\frac{2}{P Q^{2}}\left(\delta^{2} x^{2}+1\right)\right\} .
\end{aligned}
$$

Thus the numerator of $\operatorname{div} T F$ is

(7) $\mathrm{I}+\mathrm{II}=\mathrm{I}^{*}+\mathrm{I}^{* *}+\mathrm{II}$

$$
\begin{aligned}
= & \frac{1}{P^{3}}\left[x^{2}\left(f f^{\prime \prime}+f^{\prime 2}\right)-f^{2} f^{\prime 2}+\frac{Q^{2}}{A^{2}}\left(\left(f^{2}\left(f f^{\prime \prime}-1\right)-x^{2}\left(f f^{\prime \prime}+f^{\prime 2}\right)\right)\right)\right] \\
+ & \frac{1}{P^{2} Q}\left[2 \delta x^{2}\left(f f^{\prime \prime}+\left(f^{\prime}\right)^{2}\right)-2 f f^{\prime}-\delta f^{2} f^{\prime 2}\right. \\
& \left.+\frac{Q^{2}}{A^{2}}\left(-\delta f^{2}+2 f f^{\prime}+3 \delta x^{2}\right)\right] \\
+ & \frac{1}{P Q^{2}}\left[\delta^{2} x^{2}\left(f^{\prime \prime} f+\left(f^{\prime}\right)^{2}\right)-1-2 \delta f f^{\prime}+2 \frac{Q^{2}}{A^{2}}\left(\delta^{2} x^{2}+1\right)\right] \\
- & \delta Q^{-3}-\frac{1}{4} P^{-2} Q^{-2}\left(P_{x} Q_{y}-Q_{x} P_{y}\right)^{2} .
\end{aligned}
$$


We want to choose $\delta$ and $A$ to make the third bracket of the right-hand side of (7) negative. For this, substituting the expression for $Q$ in the bracket and rewriting it as

$\mathrm{III}=-1+\left(\frac{2}{A^{2}}\right)\left(y_{0}-y+\frac{\delta x^{2}}{2}\right)^{2}\left(1+\delta^{2} x^{2}\right)+\delta^{2} x^{2}\left(f^{\prime \prime} f+\left(f^{\prime}\right)^{2}\right)-2 \delta f f^{\prime}$.

For any given $\lambda, 0<\lambda<\frac{1}{4}$, if we take $\delta$ such that

$$
0<\delta<\inf _{y \in\left(0, y_{1}\right)} \min \left\{\frac{\lambda y_{0}}{f^{2}}, \frac{\lambda}{f}, \frac{\lambda}{f^{2}}, \frac{\lambda}{f\left(f^{\prime \prime} f+\left(f^{\prime}\right)^{2}\right)^{\frac{1}{2}}}\right\}
$$

and $A=4 \sqrt{2} y_{0}$, then we have

$$
\mathrm{III} \leq-1+\frac{1}{4\left(2 y_{0}\right)^{2}}\left(y_{0}+\frac{\lambda y_{0}}{2}\right)^{2}\left(1+\lambda^{2}\right)+\lambda^{2} \leq-1+\frac{1+\lambda^{2}}{4}+\lambda^{2}<0 .
$$

As of the second bracket of the right-hand side of (7), to make it negative, it clearly suffices to make the following expression negative, namely

$$
\mathrm{IV}=\left(\frac{Q^{2}}{A^{2}}\right)\left(f f^{\prime}+\frac{3}{2} \delta x^{2}\right)-f f^{\prime}+\delta x^{2}\left(f f^{\prime \prime}+\left(f^{\prime}\right)^{2}\right) .
$$

For this, we observe that, as $x^{2}<f^{2}$ in $\Omega$ and $f>0, f^{\prime}>0$ and $f^{\prime \prime}>0$ in $(0, \infty)$,

$$
\frac{3}{2} \delta x^{2}+f f^{\prime} \leq \frac{3}{2} \delta f^{2}+f f^{\prime}=f f^{\prime}\left(1+\frac{3}{2} \delta \frac{f^{2}}{f f^{\prime}}\right),
$$

while

$$
\begin{aligned}
-f f^{\prime}+\delta x^{2}\left(f f^{\prime \prime}+\left(f^{\prime}\right)^{2}\right) & \leq-f f^{\prime}+\delta f^{2}\left(f f^{\prime \prime}+\left(f^{\prime}\right)^{2}\right) \\
& =-f f^{\prime}\left(1-\delta \frac{f^{2}\left(f f^{\prime \prime}+\left(f^{\prime}\right)^{2}\right.}{f f^{\prime}}\right)
\end{aligned}
$$

and furthermore, if we require that

$$
\delta<\inf _{y \in\left(0, y_{1}\right)} \min \left\{\frac{\lambda f f^{\prime}}{f^{2}\left(f f^{\prime \prime}+\left(f^{\prime}\right)^{2}\right)}, \frac{\lambda f f^{\prime}}{2 f^{2}}\right\}
$$

it follows from (9) that

$$
\frac{Q^{2}}{A^{2}} \leq \frac{1}{4}
$$

And also, we have

$$
\mathrm{IV} \leq f f^{\prime}\left(\frac{Q^{2}}{A^{2}}(1+\lambda)+\lambda-1\right) \leq f f^{\prime}\left(\frac{1}{4}(1+\lambda)+\lambda-1\right) \leq \frac{-1}{4} f f^{\prime} .
$$

Thus, the condition that $f>0, f^{\prime}>0$ in $(0, \infty)$ ensures us of the negativity of (10). It remains to consider the first bracket of the right-hand side of (7). 
To make it negative, it suffices to make negative the following expression

$$
\mathrm{V}=x^{2}\left(f f^{\prime \prime}+\left(f^{\prime}\right)^{2}\right)-f^{2} f^{\prime 2}+\frac{Q^{2}}{A^{2}} f^{2}\left(f f^{\prime \prime}-1\right),
$$

or, in view of $(11)$,

$$
\mathrm{V} \leq x^{2}\left(f f^{\prime \prime}+\left(f^{\prime}\right)^{2}\right)-f^{2} f^{\prime 2}+\frac{1}{4} f^{2}\left(f f^{\prime \prime}\right) .
$$

Recall that for given function $f$ as above, we define

$$
p(f)=1-\frac{f f^{\prime \prime}}{\left(f^{\prime}\right)^{2}} .
$$

For $\S 5$, and from now on, we assume that $-1 \leq p(f) \leq 1$, following a remark concerning $p(f)$ for our interesting functions, [5, Remark 2.7]. And so, in particular for $f(y)=(y+z)^{m}, p(f)=\frac{1}{m}$ and for $f(y)=a e^{b y}, p(f)=0$ where $z, m>1, a$, and $b$ are positive constants, and also it is easy to see that for $f(y)=e^{y^{\alpha}}$, with $\alpha>1$, then $p(f) \longrightarrow 0^{-}$as $y \longrightarrow+\infty$.

Rewriting (12) in terms of $p(f)$, and noticing that $\left(\frac{3}{4}+\frac{p}{4}\right) \times\left(\frac{1}{2-p}\right) \geq \frac{1}{6}$, we have

$$
\mathrm{V} \leq(2-p)\left(f^{\prime}\right)^{2}\left(x^{2}-\frac{\frac{3}{4}+\frac{p}{4}}{2-p} f^{2}\right) \leq(2-p)\left(f^{\prime}\right)^{2}\left(x^{2}-\frac{1}{6} f^{2}\right),
$$

and so if we assume furthermore that

$$
\Omega \subseteq\left\{(x, y) \in \mathbb{R}^{2} \mid-\frac{1}{\sqrt{6}} f(y)<x<\frac{1}{\sqrt{6}} f(y), y>0\right\} \times \mathbb{R}^{n-2} \subseteq \mathbb{R}^{n},
$$

then $\mathrm{V} \leq 0$ and get the following conclusion about the estimation of our comparison function: If $F=\sqrt{f^{2}(y)-x^{2}} \frac{4 \sqrt{2} y_{0}}{\left(y_{0}-y+\frac{\delta}{2} x^{2}\right)}$ with $\delta$ as in our assumptions, (9), $\left(9^{*}\right)$, then $\operatorname{div} T F \leq 0$ in $\Omega_{y_{0}, \delta}$, where $\Omega$ is assumed as in (14). Now we state what we achieved as follows:

Proposition 5. Let $f_{1}:[0, \infty) \longrightarrow[0, \infty)$, and $f_{1} \in C^{2}([0, \infty))$ with $f_{1}>$ $0, f_{1}{ }^{\prime}>0, f_{1}{ }^{\prime \prime}>0$ on $[0, \infty)$, and $-1 \leq p\left(f_{1}\right) \leq 1$. Suppose that $\Omega \subseteq$ $\left\{(x, y) \in \mathbb{R}^{2} \mid-f_{1}(y)<x<f_{1}(y), y>0\right\} \times \mathbb{R}^{n-2} \subseteq \mathbb{R}^{n}$ and that $u \in$ $C^{2}(\Omega) \cap C^{0}(\bar{\Omega})$ and for some constant $\beta$ with $0<\beta<1$ satisfying

$$
\begin{cases}\operatorname{div} T u \geq 0 & \text { in } \Omega \\ u \leq 4 \sqrt{2} \beta \sqrt{6 f_{1}^{2}(y)-x^{2}} & \text { on } \partial \Omega .\end{cases}
$$

Then $u \leq 4 \sqrt{2} \sqrt{6 f_{1}^{2}(y)-x^{2}}$ in $\Omega$.

Proof. Set $f(y)=\sqrt{6} f_{1}(y)$ and define $F(x, y)=4 \sqrt{2} y_{0} \frac{\left(f^{2}(y)-x^{2}\right)^{\frac{1}{2}}}{\left(y_{0}-y+\frac{\delta}{2} x^{2}\right)}$ as above, where $y_{0}>0$ and $\delta>0$, small as in (9) and $\left(9^{*}\right)$ and we also require 
that $\delta \leq \frac{(2-2 \beta) y_{0}}{\beta\left(f\left(y_{1}\right)\right)^{2}}$. Then following the computation as above, in particular that of (7), and also noticing that the first three brackets of the right-hand side of (7) are negative in $\Omega_{y_{0}, \delta}$ as shown above, it is easy to see that

$$
\operatorname{div} T F=\frac{\left(\frac{1}{F^{2}}+\frac{F_{y}^{2}}{F^{2}}\right) \frac{F_{x x}}{F}-2 \frac{F_{x}}{F} \frac{F_{y}}{F} \frac{F_{x y}}{F}+\left(\frac{1}{F^{2}}+\frac{F_{x}^{2}}{F^{2}}\right) \frac{F_{y y}}{F}}{\frac{1}{F^{3}}\left(1+|\nabla F|^{2}\right)^{\frac{3}{2}}}
$$

and

$$
\left(\frac{1}{F^{2}}+\frac{F_{x}^{2}}{F^{2}}\right) \frac{F_{y y}}{F}-2 \frac{F_{x}}{F} \frac{F_{y}}{F} \frac{F_{x y}}{F}+\left(\frac{1}{F^{2}}+\frac{F_{y}^{2}}{F^{2}}\right) \frac{F_{x x}}{F}<-\delta\left(y_{0}-y+\frac{\delta}{2} x^{2}\right)^{-3},
$$

when $(x, y)$ is close to $\left\{(x, y) \in \mathbb{R}^{2} \mid y_{0}-y+\frac{\delta}{2} x^{2}=0\right\}$.

And so, noticing that $P=f^{2}(y)-x^{2}, Q=y_{0}-y+\frac{\delta}{2} x^{2}$ and $A=4 \sqrt{2} y_{0}$, when $(x, y)$ is close to $\left\{(x, y) \in \mathbb{R}^{2} \mid y_{0}-y+\frac{\delta}{2} x^{2}=0\right\}$, we have

$$
\begin{aligned}
\operatorname{div} T F & \leq-\delta Q^{-3}\left(\frac{1}{F^{2}}+\left|\frac{\nabla F}{F}\right|^{2}\right) \frac{-3}{2} \\
& \leq-\delta Q^{-3}\left(\frac{Q^{2}}{A^{2} P}+\left|\frac{1}{2} \frac{\nabla P}{P}-\frac{\nabla Q}{Q}\right|^{2}\right) \frac{-3}{2} \\
& \leq-\delta\left(\frac{Q^{4}}{A^{2} P}+\left|\frac{1}{2} \frac{Q}{P} \nabla P-\nabla Q\right|^{2}\right) \frac{-3}{2} \\
& \leq-\delta\left(\frac{Q^{4}}{A^{2} P}+\frac{1}{4} \frac{Q^{2}}{P^{2}}|\nabla P|^{2}+|\nabla Q|^{2}-\frac{Q}{P} \nabla P \cdot \nabla Q\right)^{\frac{-3}{2}} \\
& \leq-\frac{\delta}{2}\left(1+\delta^{2} x^{2}\right)^{\frac{-3}{2}}
\end{aligned}
$$

$$
\text { since } \frac{-Q}{P} \nabla P \cdot \nabla Q \geq 0 \text { and }|\nabla Q|^{2}=1+\delta^{2} x^{2} .
$$

But the bounded connected component of the closure of $\left\{(x, y) \in \mathbb{R}^{2} \mid-\right.$ $\left.f_{1}(y)<x<f_{1}(y), y>0\right\} \cap\left\{(x, y) \in \mathbb{R}^{2} \mid y_{0}-y+\frac{\delta}{2} x^{2}>0\right\}$, which is denoted as $\overline{\Omega^{*}}$, is compact. And we have $\Omega_{y_{0}, \delta} \subseteq \Omega^{*} \times \mathbb{R}^{n-2}$, and so, there exists a positive constant $c$, such that

$$
\begin{cases}\operatorname{div} T F \leq-c & \text { in } \Omega_{y_{0}, \delta}, \\ F \geq u & \text { on } \partial \Omega_{y_{0}, \delta} \cap\left\{(x, y) \in \mathbb{R}^{2} \mid y_{0}-y+\frac{\delta}{2} x^{2}>0\right\} \times \mathbb{R}^{n-2}, \\ F=+\infty & \text { on } \partial \Omega_{y_{0}, \delta} \cap\left\{(x, y) \in \mathbb{R}^{2} \mid y_{0}-y+\frac{\delta}{2} x^{2}=0\right\} \times \mathbb{R}^{n-2} .\end{cases}
$$

Now, by Lemma 4 , we have $u \leq F$ in $\Omega_{y_{0}, \delta}$, which is

$$
u\left(x, y, z_{1}, \ldots, z_{n-2}\right) \leq 4 \sqrt{2} y_{0} \frac{\left(6 f_{1}^{2}(y)-x^{2}\right)^{\frac{1}{2}}}{\left(y_{0}-y+\frac{\delta}{2} x^{2}\right)} \quad \text { in } \Omega_{y_{0}, \delta} .
$$


Now, let $\delta \longrightarrow 0$ and then let $y_{0} \longrightarrow+\infty$, we get the conclusion of the proof.

\section{Phragmèn-Lindelöf theorem in higher dimensions.}

First, let's generalize Theorem 2 as follows:

Theorem 6. Let $\Omega \subseteq\left\{(x, y) \in \mathbb{R}^{2} \mid-a y^{m}<x<a y^{m}, y>0\right\} \times \mathbb{R}^{n-2} \subseteq \mathbb{R}^{n}$ be an unbounded domain, where $m \geq 1$ and a are positive constants. Let $u \in C^{2}(\Omega) \cap C^{0}(\bar{\Omega})$ and suppose that

$$
\begin{cases}\operatorname{div} T u \geq 0 & \text { in } \Omega \\ u \leq a y^{m} h_{m}\left(\frac{x}{a y^{m}}\right) & \text { on } \partial \Omega .\end{cases}
$$

Then we have $u \leq a y^{m} h_{m}\left(\frac{x}{a y^{m}}\right) \leq a y^{m} h_{\infty}\left(\frac{x}{a y^{m}}\right)=\sqrt{a^{2} y^{2 m}-x^{2}}$ in $\Omega$.

Proof. For every given positive constant $\epsilon>0$, we now set $f_{\epsilon}(x, y)=$ $a(y+\epsilon)^{m+\epsilon}, F_{\epsilon}\left(x, y, z_{1}, z_{2}, \ldots, z_{n-2}\right)=a(y+\epsilon)^{m+\epsilon} h_{m+\epsilon}\left(\frac{x}{a(y+\epsilon)^{m+\epsilon}}\right)$, where $\left(x, y, z_{1}, z_{2}, \ldots, z_{n-2}\right) \in \Omega$.

Since $-a y^{m}<x<a y^{m}, y>0$, we have

$$
\left|\frac{x}{a(y+\epsilon)^{m+\epsilon}}\right| \leq \frac{y^{m}}{(y+\epsilon)^{m+\epsilon}} \longrightarrow 0 \quad \text { as } y \longrightarrow+\infty \text {. }
$$

By Lemma $1, h_{m+\epsilon}\left(\frac{x}{a(y+\epsilon)^{m+\epsilon}}\right) \longrightarrow h_{m+\epsilon}(0)$ uniformly as $y \longrightarrow+\infty$, and so it is easy to see that there exists a large constant $y_{3}$ such that $F_{\epsilon}(x, y) \geq$ $\sqrt{200 a^{2} y^{2 m}-x^{2}}$ for $y \geq y_{3}$.

Next by [6, Theorem 2], setting $f_{\epsilon}(y)=a(y+\epsilon)^{m+\epsilon}, t=\frac{x}{f_{\epsilon}(y)}$ and recalling that $p\left(f_{\epsilon}\right)=\frac{1}{m+\epsilon}$, we have

$\operatorname{div} T F_{\epsilon}$

$$
\begin{aligned}
= & \left(1+\left|\nabla F_{\epsilon}\right|^{2}\right)^{-\frac{3}{2}} \frac{\left(f_{\epsilon}^{\prime}\right)^{2}}{f_{\epsilon}} \\
& \cdot\left(\left(1-p\left(f_{\epsilon}\right)\right)\left(h_{m+\epsilon}-t h_{m+\epsilon}^{\prime}\right)\left(\left(h_{m+\epsilon}^{\prime}\right)^{2}+1\right)+h_{m+\epsilon}^{\prime \prime}\left(h_{m+\epsilon}^{2}+t^{2}\right)+\frac{h_{m+\epsilon}^{\prime \prime}}{\left(f_{\epsilon}^{\prime}\right)^{2}}\right) .
\end{aligned}
$$

Since $h_{m+\epsilon}(t)$ is the solution of $(*)$ and $(* *)$ with $p\left(f_{\epsilon}\right)=\frac{1}{m+\epsilon}$, we have

$$
\operatorname{div} T F_{\epsilon}=\left(1+\left|\nabla F_{\epsilon}\right|^{2}\right)^{-\frac{3}{2}} \frac{\left(f_{\epsilon}^{\prime}\right)^{2}}{f_{\epsilon}} \cdot \frac{h_{m+\epsilon}^{\prime \prime}}{\left(f_{\epsilon}^{\prime}\right)^{2}}
$$

and so obviously that $\operatorname{div} T F_{\epsilon}<0$ on $\overline{\Omega^{\prime}}$ where $\Omega^{\prime}=\Omega \cap\left\{\left(x, y, z_{1}, \ldots, z_{n-2}\right)\right.$ $\left.\in \mathbb{R}^{n} \mid 0<y<y_{3}\right\}$.

And so, there exists a positive constant $C_{1}>0$ such that

$$
\operatorname{div} T F_{\epsilon} \leq-C_{1} \quad \text { on } \overline{\Omega^{\prime}} .
$$


But, noticing that

$$
u \leq a y^{m} h_{m}\left(\frac{x}{a y^{m}}\right) \leq \sqrt{a^{2} y^{2 m}-x^{2}} \leq 4 \sqrt{2} \beta \sqrt{6 a^{2} y^{2 m}-x^{2}},
$$

for some constant $\beta<1$ on $\partial \Omega$, by Proposition 5 , we also have

$$
u \leq 4 \sqrt{2} \sqrt{6 a^{2} y^{2 m}-x^{2}} \leq \sqrt{200 \cdot a^{2} y^{2 m}-x^{2}} \quad \text { in } \Omega \backslash \Omega^{\prime} .
$$

By Lemma 4, we have

$$
u \leq F_{\epsilon} \quad \text { in } \quad \overline{\Omega^{\prime}} .
$$

In conclusion, we have

$$
u \leq F_{\epsilon} \quad \text { in } \Omega,
$$

and let $\epsilon \longrightarrow 0$, the proof is done.

As a corollary of Theorem 6, we state a generalization of Nitsche's theorem [7] as follows.

Corollary. Let $\Omega=\left\{(x, y) \in \mathbb{R}^{2} \mid-\right.$ ay $\left.<x<a y, y>0\right\} \times \mathbb{R}^{n-2}$ be a wedge domain, where $a$ is a positive constant. Let $u \in C^{2}(\Omega) \cap C^{0}(\bar{\Omega})$ and suppose that

$$
\begin{cases}\operatorname{div} T u=0 & \text { in } \Omega, \\ u=0 & \text { on } \partial \Omega .\end{cases}
$$

Then $u \equiv 0$ in $\Omega$.

Proof. Apply Theorem 6 to functions $u$ and $-u$, we have $u \leq 0$ in $\Omega$ and $-u \leq 0$ in $\Omega$, and so $u \equiv 0$ as claimed.

Next, let's generalize Theorem $2^{*}$ as follows:

Theorem 6*. Let $\Omega \subseteq\left\{(x, y) \in \mathbb{R}^{2} \mid-a e^{b y}<x<a e^{b y}, y>0\right\} \times \mathbb{R}^{n-2} \subseteq$ $\mathbb{R}^{n}$, where $a, b$ are positive constants. Let $u \in C^{2}(\Omega) \cap C^{0}(\bar{\Omega})$ and suppose that

$$
\begin{cases}\operatorname{div} T u \geq 0 & \text { in } \Omega \\ u \leq \sqrt{a^{2} e^{2 b y}-x^{2}} & \text { on } \partial \Omega .\end{cases}
$$

Then we have $u \leq \sqrt{a^{2} e^{2 b y}-x^{2}}$ in $\Omega$.

Proof. The proof is similar to that of Theorem 6 .

For every $\epsilon>0$, we consider the following function

$$
F_{\epsilon}\left(x, y, z_{1}, z_{2}, \ldots, z_{n-2}\right)=a e^{(b+\epsilon) y} h_{\infty}\left(\frac{x}{a e^{(b+\epsilon) y}}\right)=\sqrt{a^{2} e^{2(b+\epsilon) y}-x^{2}}
$$

with $\left(x, y, z_{1}, \ldots, z_{n-2}\right) \in \Omega$.

Since $-a e^{b y}<x<a e^{b y}, y>0$, we have

$$
\left|\frac{x}{a e^{(b+\epsilon) y}}\right| \leq \frac{a e^{b y}}{a e^{(b+\epsilon) y}} \longrightarrow 0 \quad \text { as } y \longrightarrow+\infty
$$

and notice that $F_{\epsilon}=a e^{(b+\epsilon) y}\left(1-\frac{x^{2}}{a^{2} e^{2(b+\epsilon) y}}\right)^{\frac{1}{2}}$. 
Hence, there exists a positive constant $y_{3}>0$ such that

$$
F_{\epsilon} \geq \sqrt{200 a^{2} e^{2 b y}-x^{2}} \quad \text { for } y \geq y_{3} .
$$

Next, by [6, Theorem 2], and setting $f_{\epsilon}(y)=a e^{(b+\epsilon) y}, t=\frac{x}{f_{\epsilon}(y)}$ and noticing that $p\left(f_{\epsilon}\right)=0$, we have

$$
\begin{aligned}
\operatorname{div} T F_{\epsilon}= & \left(1+\left|\nabla F_{\epsilon}\right|^{2}\right)^{-\frac{3}{2}} \frac{\left(f_{\epsilon}^{\prime}\right)^{2}}{f_{\epsilon}} \\
& \cdot\left(\left(h_{\infty}-t h_{\infty}^{\prime}\right)\left(h_{\infty}^{\prime}{ }^{2}+1\right)+h_{\infty}^{\prime \prime}\left(h_{\infty}^{2}+t^{2}\right)+\frac{h_{\infty}^{\prime \prime}}{f_{\epsilon}^{\prime 2}}\right) \\
= & \left(1+\left|\nabla F_{\epsilon}\right|^{2}\right)^{-\frac{3}{2}} \frac{\left(f_{\epsilon}^{\prime}\right)^{2}}{f_{\epsilon}} \frac{h_{\infty}^{\prime \prime}}{\left(f_{\epsilon}^{\prime}\right)^{2}} .
\end{aligned}
$$

So, we have

$$
\operatorname{div} T F_{\epsilon}<0 \quad \text { on } \overline{\Omega^{\prime}},
$$

where

$$
\Omega^{\prime}=\Omega \cap\left\{\left(x, y, z_{1}, \ldots, z_{n-2}\right) \in \mathbb{R}^{n} \mid \quad 0<y<y_{3}\right\},
$$

and so there exists a positive constant $C_{1}>0$ such that

$$
\operatorname{div} T F_{\epsilon} \leq-C_{1} \quad \text { in } \overline{\Omega^{\prime}} .
$$

Finally, by Proposition 5, notice that

$$
u \leq \sqrt{a^{2} e^{2 b y}-x^{2}} \leq 4 \sqrt{2} \beta \sqrt{6 a^{2} e^{2 b y}-x^{2}},
$$

for some constant $\beta<1$ on $\partial \Omega$, we also have

$$
u \leq 4 \sqrt{2} \sqrt{6 a^{2} e^{2 b y}-x^{2}} \leq F_{\epsilon} \quad \text { in } \Omega \backslash \Omega^{\prime} .
$$

So, by Lemma 4, we have

$$
u \leq F_{\epsilon} \quad \text { on } \overline{\Omega^{\prime}},
$$

and so obviously, we get

$$
u \leq F_{\epsilon} \quad \text { in } \Omega,
$$

and let $\epsilon \longrightarrow 0$, the proof is finished.

Finally, let's generalize Theorem 3 as follows:

Theorem 7. Let $f_{1} \in C^{2}([0, \infty))$ with $f_{1} \geq 0, f_{1}^{\prime}>0$, and $f_{1}^{\prime \prime} \geq 0$ in $(0, \infty)$ such that $p\left(f_{1}\right) \geq p_{0}$, where $p_{0}$ is a constant with $-1 \leq p_{0} \leq 0$. Suppose that $\Omega \subseteq\left\{(x, y) \in \mathbb{R}^{2} \mid \quad-f_{1}(y)<x<f_{1}(y), y>0\right\} \times \mathbb{R}^{n-2} \subseteq \mathbb{R}^{n}$ and $u \in C^{2}(\bar{\Omega}) \cap C^{0}(\bar{\Omega})$ satisfying

$$
\begin{cases}\operatorname{div} T u \geq 0 & \text { in } \Omega \\ u \leq a \sqrt{f^{2}(y)-x^{2}} & \text { on } \partial \Omega,\end{cases}
$$

where $f=\left(\frac{\left(a^{2}-1\right)\left(2-p_{0}\right)}{\left(a^{2}-1+p_{0}\right)}\right)^{\frac{1}{2}} f_{1}$ and $a$ is a positive constant with $a^{2}-1+p_{0}>0$.

Then we have $u \leq a \sqrt{f^{2}(y)-x^{2}}$ in $\Omega$. 
Proof. For any given $\epsilon>0$, we define $f_{\epsilon}(y)=e^{\epsilon y} f(y+\epsilon)$ and $F_{\epsilon}\left(x, y, z_{1}, \ldots\right.$, $\left.z_{n-2}\right)=a \sqrt{f_{\epsilon}^{2}(y)-x^{2}}$, then there exists $y_{3}>0$ such that

$$
F_{\epsilon} \geq a\left(e^{2 \epsilon y} f^{2}(y)-x^{2}\right)^{\frac{1}{2}} \geq\left(200 \cdot a^{2} f^{2}(y)-x^{2}\right)^{\frac{1}{2}} \quad \text { for } \quad y>y_{3} .
$$

Computing the mean curvature of $F_{\epsilon}$ and using the definition, $p\left(f_{\epsilon}\right)=1-$ $\frac{f_{\epsilon} f_{\epsilon}^{\prime \prime}}{\left(f_{\epsilon}^{\prime}\right)^{2}}$, we have

$$
\begin{aligned}
\operatorname{div} T F_{\epsilon}=(1 & \left.+\left|\nabla F_{\epsilon}\right|^{2}\right)^{-\frac{3}{2}}\left(f_{\epsilon}^{2}-x^{2}\right)^{\frac{-3}{2}} \\
& \cdot\left(a\left(f_{\epsilon}^{\prime}\right)^{2}\left[\left(a^{2}-1\right)\left(2-p\left(f_{\epsilon}\right)\right) x^{2}-f_{\epsilon}^{2}\left(a^{2}-1+p\left(f_{\epsilon}\right)\right)\right]-a f_{\epsilon}^{2}\right) .
\end{aligned}
$$

Obviously, we have

$$
f_{\epsilon}^{2}(y) \geq f^{2}(y) \geq \frac{\left(a^{2}-1\right)\left(2-p_{0}\right)}{\left(a^{2}-1+p_{0}\right)} f_{1}^{2}(y) \geq \frac{\left(a^{2}-1\right)\left(2-p\left(f_{\epsilon}\right)\right)}{\left(a^{2}-1+p\left(f_{\epsilon}\right)\right)} f_{1}^{2}(y),
$$

and so, we have

$$
\operatorname{div} T F_{\epsilon} \leq-a\left(1+\left|\nabla F_{\epsilon}\right|^{2}\right)^{-\frac{3}{2}} f_{\epsilon}^{2}<0 \quad \text { in } \Omega,
$$

and by compactness, there exists a positive constant $C_{1}>0$ such that

$\operatorname{div} T F_{\epsilon} \leq-C_{1} \quad$ in $\Omega_{1}=\left\{\left(x, y, z_{1}, z_{2}, \ldots, z_{n-2}\right) \in \Omega \mid y<y_{3}\right\}$.

But by Proposition 5, notice that

$$
u \leq a \sqrt{f^{2}(y)-x^{2}} \leq 4 \sqrt{2} \beta \sqrt{6 a^{2} f^{2}-x^{2}},
$$

for some constant $\beta<1$ on $\partial \Omega$, we also have

$$
u \leq \sqrt{200 a^{2} f^{2}(y)-x^{2}} \leq F_{\epsilon} \quad \text { in } \Omega \backslash \Omega_{1} .
$$

By Lemma 4, we have

$$
u \leq F_{\epsilon} \quad \text { in } \Omega_{1} .
$$

In conclusion, we have $u \leq F_{\epsilon}$ in $\Omega$, and then let $\epsilon \longrightarrow \infty$.

We thus finish the proof.

\section{References}

[1] R. Finn, Equilibrium Capillary Surfaces, Springer-Verlag, New York-Berlin-Heidelberg-Tokyo, 1986, MR 88f:49001, Zbl 0583.35002.

[2] R. Finn and J. Hwang, On the comparison principle for capillary surfaces, J. Fac. Sci. Univ. Tokyo Sect. IA, Math., 36 (1989), 131-134, MR 90h:35099, Zbl 0684.35007.

[3] D. Gilbarg and N.S. Trudinger, Elliptic Partial Differential Equations of Second Order, Second edition, Springer-Verlag, Berlin-Heidelberg-New York-Tokyo, 1983, MR 86c:35035, Zbl 0562.35001.

[4] J. Hwang, Phragmèn-Lindelöf theorem for the minimal surface equation, Proc. Amer. Math. Soc., 104 (1988), 825-828, MR 89j:35016, Zbl 0787.35012.

[5] _ Growth property for the minimal surface equation in unbounded domains, Proc. Amer. Math. Soc., 121 (1994), 1027-1037, MR 94j:35019, Zbl 0820.35010. 
[6] _ Catenoid-like solutions for the minimal surface equation, Pacific J. Math., 183 (1998), 91-102, MR 99d:58041, Zbl 0905.35029.

[7] J.C.C. Nitsche, On new results in the theory of minimal surface, Bull. Amer. Math. Soc., 71 (1965), 195-270, MR 30 \#4200, Zbl 0135.21701.

[8] _ , Vorlesungen über Minimalflächen, Springer-Verlag, Berlin-Heidelbert-New York, 1975, MR 56 \#6533, Zbl 0319.53003.

[9] R. Osserman, A Survey of Minimal Surfaces, Van Nostrand-Reinhold, New York, 1969, MR 41 \#934, Zbl 0209.52901.

[10] M.H. Protter and H.F. Weinberger, Maximum Principles in Differential Equations, Prentice-Hall, Englewood Cliffs, N.J., 1967, MR 36 \#2935, Zbl 0153.13602.

Received February 27, 2001.

Mathematics Department

Academia Sinica

NAN-KANG

TAIPEI, 115, TAIWAN

E-mail address: macchsieh@ccvax.sinica.edu.tw

Mathematics Department

ACADEMIa Sinica

NAN-KANG

TAIPEI, 115, TAIWAN

E-mail address: majfh@ccvax.sinica.edu.tw

Mathematics Department

Academia Sinica

NAN-KANG

TAIPEI, 115, TAIWAN

E-mail address: liang@math.sinica.edu.tw 